Voix et Images

voixetimages

\title{
Cryptes et révélations
}

\section{Lucie Robert}

Volume 27, numéro 2 (80), hiver 2002

La sociabilité littéraire

URI : https://id.erudit.org/iderudit/290063ar

DOI : https://doi.org/10.7202/290063ar

Aller au sommaire du numéro

\section{Éditeur(s)}

Université du Québec à Montréal

\section{ISSN}

0318-9201 (imprimé)

1705-933X (numérique)

Découvrir la revue

\section{Citer cet article}

Robert, L. (2002). Cryptes et révélations. Voix et Images, 27(2), 353-360.

https://doi.org/10.7202/290063ar d'utilisation que vous pouvez consulter en ligne.

https://apropos.erudit.org/fr/usagers/politique-dutilisation/ 


\section{Dramaturgie}

\section{Cryptes et révélations}

\section{Lucie Robert, Université du Québec à Montréal}

Techniquement, le cryptage suggère l'inscription d'un code dans un autre. Il s'agit donc d'un procédé de codage qui a pour but de masquer à l'œil non informé ou moins attentif le sens même du message. La crypte est aussi un caveau souterrain. Ce qu'elle suggère ainsi est l'existence de deux plans d'action ou de récit. L'un qui paraît à l'oeil nu, en surface, sur scène; l'autre, qu'on ne verra pas, mais qui détermine la première. Elle suscite parfois la frustration du spectateur qui ne possédera peut-être même jamais la clé de l'énigme. Au cryptage répond le décryptage, c'està-dire une forme de révélation qui tient moins au récit explicatif que ferait un personnage qu'à la mise au jour d'une image latente. Si la révélation est courante au théâtre, bien qu'elle y apparaisse surtout sous la forme d'un récit qui entraîne le dénouement, le cryptage l'est moins. 
Que les auteurs dramatiques tendent à remplacer l'action dramatique par la mise en scène d'une énigme, est un autre indice du caractère prépondérant de la narration sur la dramatisation dans la dramaturgie contemporaine. Et si les auteurs dramatiques dont il est question ici sont aussi, pour la plupart, des romanciers, la chose ne devrait pas nous étonner: confronté à l'indicible, le romancier doit se taire ou trouver une autre manière de dire. Or, cacher, ne pas montrer reste le privilège d'un art qui repose précisément sur le visible. En passant d'une écriture narrative à une écriture dramatique, l'auteur renforce son propos. Encore faut-il qu'il en ait eu un au départ.

Normand Chaurette excelle dans ce processus qui consiste à multiplier les strates d'action dramatique, soit par enchâssement, soit par cryptage, parfois. les deux à la fois. Et c'est bien le raffinement qu'il atteint dans ce type de structure qui séduit tant les amateurs de thêâtre, pourtant fréquemment rebutés par le caractère statique de l'action dramatique ou carrément par son absence ou plutôt par sa dissolution progressive. On se rappellera la variante du tableau 6 dans Provincetoun Playbouse, juillet 1919, j'avais 19 ans, l'encre sympathique des Fragments d'une lettre d'adieu lus par des géologues, l'anecdote en creux de Stabat Mater II. De même, chacune de ces pièces insère au cœur de l'action un autre texte que nous ne connaissons que par fragments. Il y a, chez Chaurette, cette inscription d'un récit impossible, peut-être même indicible, d'un roman, d'une pièce, d'un poème, d'une lettre qu'on n'écrira pas jusqu'à la fin et dont le propos reste une énigme: le Thêatre de l'immolation de la beauté dans Provincetoun Playbouse, la lettre d'adieu elle-même, le roman de la neuvième mère de Stabat Mater II. Et l'on pourrait multiplier les exemples par l'analyse de chaque pièce, depuis la toute première, Rêve d'une nuit d'hôpital, qui emprunte son titre à un poème de Nelligan, jusqu'au Passage de l'Indiana qui pousse cette logique au bout d'elle-même. Pour cette raison, l'appel au décryptage, il y a souvent un plus grand plaisir à lire une pièce de Chaurette qu'à la voir, quel que soit par ailleurs le talent de la mise en scène.

L'inscription de la musique, comme une forme structurant l'action dramatique, depuis Stabat Mater $I I^{1}$, introduit une motivation nouvelle au cryptage, en reformulant le problème de l'action dramatique. Il ne s'agit plus de révéler l'action cryptée comme une énigme, mais de révéler son caractère indicible et de substituer ainsi, à l'action elle-même, les sensations qu'elle produit ou les conséquences qu'elle engendre, car "même au thêâtre, un enfant ne [peut] être éventré sans susciter quelque rumeur " ". Or, de Provincetoun Playbouse jusqu'à Stabat Mater II et Le petit Köchel, l'événement indicible concerne bien la mort d'un enfant. Comme l'écrit l'auteure de L'enfant du désert, la romancière de Stabat Mater II: " Il y a de l'indécence à publier ce genre d'histoire dans une ville comme Manustro. Il suffisait d'y penser. Tout le monde y trouve son compte, à commencer par l'éditeur " (p. 31). Ce roman, bien entendu, on ne le lira pas autrement que par de rares fragments. Ce qu'on lira d'abord est la douleur éprouvée par les 
mères, qui forment un chœur de dix neuf voix, comme autant de variations autour d'un thème unique, dont l'organisation emprunte une forme musicale canonique.

Tout aussi indicible est l'action cryptée dans Le petit Köchel ${ }^{3}$, pièce créée au Festival d'Avignon le 8 juillet 2000, dans une mise en scène de Denis Marleau pour le théâtre UBU et reprise à Montréal au Thêâtre d'Aujourd'hui au cours de la saison suivante. En ce soir du 31 octobre, soir de l'Halloween, qui, cette année-là, est le dernier samedi du mois, c'est-à-dire le jour d'automne où l'on doit reculer l'heure, un événement doit se produire: "Il a parlé. Notre enfant, Cécile, notre fils: pour la première fois depuis qu'il s'est terré dans la cave, il a dit, je te le jure, il a dit: "C'est aujourd'hui que j'ai décidé de me pendre." (p. 13) L'annonce est extraordinaire, on le conçoit. D'autant que le fils émet d'étranges conditions à la réalisation de sa promesse: "ses" mères, les soeurs Motherwell (Lili et Cécile, pianistes) et les sœurs Brunswick (Irène et Anne, musicologues), devront d'abord le dévorer puis commémorer l'événement chaque année par la suite. Aussi la pièce de Chaurette n'est-elle pas sans rappeler La nuit de la grande citrouille de Victor-Lévy Beaulieu, pièce connue aussi sous le titre de Votre fille Peuplesse, par inadvertance. Réunir ces deux auteurs dramatiques ne va pas de soi et pourtant, le caractère grotesque et répétitif de l'action dramatique conçue par Chaurette et le moment où elle se passe ne sont pas sans faire écho à l'œuvre de Beaulieu. Ce qui distingue Le petit Köchel est toutefois le fait que, de cette "lamentable histoire" (p. 33), nous ne verrons rien.

Ce que nous voyons contribue au contraire à déconstruire le caractère singulier de l'événement annoncé. Certaines répliques se répètent "As-tu reculé l'heure?"; "Retour en si bémol"); d'autres semblent arriver à un moment inopportun ("Vous venez encore de devancer une réplique importante, p. 20); certaines autres paraissent d'une authenticité douteuse ("Vous ajoutez des répliques! Vous n'avez pas le droit. [...] Vous improvisez!", p. 44). Peu à peu, l'évidence s'impose: loin d'être singulier, l'événement n'est qu'une répétition, et même une répétition usée, avec "trop de politesse et de pureté mais trop peu de passion" (p. 24). Si le fils avait exigé, avant de se pendre, que ses mères répètent la scène chaque soir, puis, après négociation, avait fini par accepter une commémoration annuelle, ce n'était pas sans avoir songé au préalable que, ce dernier samedi d'octobre, il fallait reculer l'heure et que, par conséquent, la scène se répéterait ad infinitum, coinçant les quatre femmes dans un temps fixe et sans issue. Pire, les mères sont peut-être déjà mortes ("Depuis que nous sommes mortes", p. 12) et peut-être ont-elles été remplacées depuis lors par des actrices qui jouent la même pièce, mais avec moins de brio, puisque la performance est entachée de fausses notes. Ainsi, aucun des événements annoncés n'aura lieu, puisque chaque fois, l'heure sera reculée: Anne ne quittera pas sa sœur; demain "la vie [ne reprendra pas] son cours" (p. 39). Plus jamais les sours Motherwell ne se consacreront à leur saison musicale.

Mais que sait-on de ce monstre? "Il disait que si l'on peut répéter 
Mozart jour après jour, on peut aussi bien rejouer la mort d'un enfant tous les soirs." (p. 50) S'est-il vraiment pendu? Car se pendre est contraire à sa nature: "Il ne supporte pas l'aboutissement. Il n'est tranquille que dans le recommencement perpétuel" (p. 39). On le présente comme un ogre, qui, deux jours plus tôt, aurait dévoré les jumelles Heifetz. Nous sommes un soir d'Halloween et ce fait seul devrait soulever quelques doutes. Il reste que les mères paraissent bien condamnées à la répétition perpétuelle. Seul espoir d'une possible transformation, Le petit Köchel lui-même, mémoire des ouvres de Mozart, dont on brûle chaque soir une page avant de reculer l'heure. Qu'arrivera-t-il quand toutes les pages auront été brûlées? Allégorie du temps qui ne passe plus, la pièce de Chaurette est aussi une réflexion sur la pérennité d'une œuvre de création : c'est en effet par sa performance sans cesse répétée que l'œuvre musicale de Mozart perdure tout comme la volonté du fils était de perpétuer sa mémoire propre. Pièce d'une structure admirable, autant que raffinée, Le Petit Köcbel n'est pas sans rappeler, par là même, Provincetown Playbouse, bien qu'elle soit peut-être moins théâtrale. Mais on comprend aisément que, dans sa version écrite, la pièce ait obtenu le Prix du Gouverneur général.

$$
\text { *** }
$$

Montrer l'indicible à travers la musique est également l'objectif que s'est fixé Gaétan Soucy qui inaugure son ouvre dramatique avec Catoblépas $^{4}$ : "[La musique] a justement pour fin de rendre sensible l'indicible, ce qui est peut-être la finalité de toute entreprise artistique d'expression. Elle est la figure de l'inexprimable, dans mes livres à tout le moins, et l'ambition de mes livres serait justement d'atteindre à la suggestion de ce qui ne saurait s'exprimer" (p. 75). Ainsi s'exprime l'auteur dans l'entrevue qu'il accordait à Stéphanie Jasmin, conseillère artistique du théâtre Ubu, et publiée à la suite de la pièce. On comprend dès lors l'intérêt de Denis Marleau pour cette pièce, étrangement proche de la précédente, et dont il a assuré également la mise en scène. La pièce a été créée le 2 mai 2001 au Studio-théâtre du Centre national des arts à Ottawa, reprise à Montréal, au Théâtre d'Aujourd'hui, le 11 septembre puis à Paris, au Théâtre de la Colline, le 9 novembre de la même année.

La pièce offre une sorte de prolongement à La petite fille qui aimait trop les allumettes. Elle met en scène deux personnages, Alice et la religieuse. "Lui, on ne peut le voir." (p. 11) Lui, c'est le fils, Robert, une sorte de monstre qui avait été enlevé à sa mère à la naissance et qu'Alice réclame comme son fils perdu. Élevé comme un orphelin, confiné à l'univers fermé de l'hôpital, "il fait de la recherche dans les sons [...] Que voulez-vous, explique la religieuse, une enfance passée à écouter la rumeur à travers les murs d'une chambre, ça finit par former l'oreille. (p. 15). Cette oreille est remarquablement fine: les premiers mots que Robert dira à sa mère, qui vient d'échapper une petite bague sur le plancher du studio, sont "Un si bémol "(p. 18). On ignorera jusqu'à la fin la nature du mal dont souffre Robert. Une seule et brève descrip- 
tion permet de caractériser son corps: Catoblépas. Ce nom, qui donne son titre à la pièce, est le nom de ce qui paraît être une poupée, une marionnette, un mannequin, "enveloppé dans une longue mante noire, avec un chapeau, et puis un masque, du genre qu'on voit au carnaval de Venise" (p. 40) et qui était en réalité le corps vivant, mais meurtri et caché, de Robert, observant de ce point de fuite les enfants, en particulier les petites filles, venues jouer là. L'image est forte, mais elle reste à peu près sans effet sur la tension dramatique, créée par l'opposition des deux femmes, seules en scène, qui se disputent la mémoire et l'affection de ce fils. "Vous ne répondez pas à ma question" (p. 12), proteste Alice d'entrée de jeu. "J'ai oublié votre question ", répond la religieuse. Car, venues pour savoir, les deux femmes oublient progressivement leur quête, comme nous aurons, nous aussi, bientôt oublié la question, voire Robert lui-même. Alice est tout ce qui compte. Sa révélation, qui clôt la pièce, concerne le récit de la mort de sa fille Ghislaine, et elle ne brille pas d'originalité. L'enfant était malade et sa mère l'a étouffée pour qu'elle cesse de souffrir. L'indicible reste inédit: de Robert et de sa musique, on ne saura rien. Il ne saurait y avoir une crypte là où n'existe aucune possibilité de décryptage. Quant à la révélation, elle n'est elle-même pas nouvelle, ayant déjà été faite vingt ans plus tôt au moment du procès, au terme duquel le juge avait prolongé l'internement d'Alice. Quel est donc alors le propos de la pièce? Crypter son propos ou le perdre de vue sont deux choses différentes.

\section{**}

L'œuvre de Stefan Psenak est peu connue au Québec, et c'est bien à tort. La fuite comme un voyage ${ }^{5}$ est sa deuxième pièce. Elle a été créée le 27 mars 2001 à La Nouvelle Scène (Ottawa), par le Théâtre du Trillium, en coproduction avec le Théâtre la Seizième et le Théâtre Français du Centre National des Arts, dans une mise en scène de Sylvie Dufour. La pièce clôt ce que l'auteur appelle son "cycle portugais", cycle entrepris avec Du chaos et de l'ordre des choses (recueil de poésie, 1998) et poursuivi avec son roman Les corps en sursis (1998) puis sa nouvelle "Le portrait inachevé" (Exister, 2001). Divisée en quinze tableaux (avec prologue et épilogue), présentés dans un ordre dont la chronologie est bouleversée, la pièce superpose deux valeurs du mot fuir. Fuir, c'est d'abord chercher un point de fuite. Aussi la pièce annonce-t-elle déjà la mise en abîme cles regards à travers l'imaginaire de l'art pictural : "[...] j'ai commencé à l'observer qui observait le monde" (p. 13), dit d'entrée de jeu Émile à propos du personnage principal, Inès. Fuir c'est aussi partir, attraper le train qui s'apprête à quitter cette gare où l'on vient tout juste de rencontrer Inès. Personnage principal, cette Inès, une artiste, agit elle-même comme point de fuite, attirant les regards et la quête des personnages masculins, et elle est en même temps celle qui fuit. Inès est un prénom lourd de sens: on pense à la reine morte de Montherlant, mais aussi à la véritable Inès de Castro, personnage emblématique de l'histoire portugaise à l'époque classique. À ce prénom répond celui de son père, Vasco 
(comme de Gama), mais répondent aussi les références à la poésie de Fernando Pessoa, à la musique du fado et au vin de Porto. En face du domaine portugais, se trouve l'Amérique, lieu du présent. Car Inès rêve de New York: "C'est là que ça se passe" (p. 25). Entre les deux, il y a "ici", le Québec ou le Canada français, rien n'est clair.

Comme Jean-Marc Dalpé, Michel Ouellette et plusieurs autres dramaturges franco-ontariens, Psenak réfère à un passé historique peu glorieux, qui renvoie à une trahison commise par le père, faute dont les enfants portent les séquelles. Ici, la référence sera portugaise: Vasco n'aurait conservé son poste d'universitaire que parce qu'il livait à la police de Salazar des renseignements sur les activités révolutionnaires de ses collègues. Contraint à l'exil par la révolution des oillets, il est venu oublier en Amérique et s'y cacher sous une nouvelle identité. Mais est-ce bien vrai? Ne serait-ce pas là plutôt un autre des mythes personnels que crée Inès, laquelle s'est déjà fait passer pour morte aux yeux de Gabriel? Comme elle a déjà prétendu que ses parents étaient retournés vivre au Portugal? Inès crypte sa quête dans son ouvre et $y$ inscrit ses deux amants aux noms d'archange, Raphaël et Gabriel. Elle écrit Raphaël, le guérisseur, et peint Gabriel, le prophète. Le manuscrit reste inédit, avant d'être finalement brûlé; le tableau est exposé en plusieurs toiles. C'est en décryptant l'œuvre d'Inès, que les archanges finissent par comprendre sa démarche: alors que Raphaël renonce à Inès, Gabriel la rejoint au Portugal, où elle est rentrée vivre une vie nou- velle. "Elle a refait le chemin en sens inverse et mis un terme à sa fuite en avant. "(p. 95) Elle a repris le vrai nom de son père, Chavez (le nom d'avant l'exil), mais elle signe toujours de son seul prénom: "la mort me va à ravir, vous ne trouvez pas?" (p. 97) Le cycle portugais ne pouvait donc pas se poursuivre, puisque Inès est retournée là-bas, assumer sa propre identité. La fuite comme un voyage, dont l'esthétique, aux dires mêmes de l'auteur, se situe à la croisée de l'écriture de Gaston Miron et de Fernando Pessoa et de la musique de Chet Baker, offre ainsi une double réflexion sur l'identité. À la question de l'identité personnelle, inscrite dans le voyage d'Inès entre ses deux pays d'appartenance, la pièce superpose la question de l'identité artistique sous la forme d'un autre voyage, entre le mot et l'image. Ici encore, le voyage arrive à son terme: "Chaque geste qu'on pose transforme un peu le monde qui nous entoure. Je veux me servir de mon coeur et de mes mains. Je veux peindre. Pour redonner un peu de beauté au monde. Parce que peindre, c'est comme aimer: c'est donner à la tristesse des airs de fête" (p. 51).

$$
* * *
$$

Créée à Radio-Québec en 1978, en version dialoguée, avec Huguette Oligny dans le rôle-titre, La dame de cent $a n s^{6}$ est publiée ici dans sa version monologue, jouée à l'Atelier l'Écart de Longueuil, le 6 août 1999, par la même comédienne. Dans l'édition qu'en a publiée Lanctôt éditeur, le monologue est suivi d'un texte plus ancien, Diogène 1960, d'abord paru dans Châtelaine en janvier 
1961. Il s'agit de la nouvelle qui a servi de point de départ au téléfilm Un cri qui vient de loin, diffusé à Radio-Canada en 1966. Conçu d'abord comme un monologue intérieur, Diogène a été présenté à haute voix, en première partie de La dame de cent ans, toujours par Huguette Oligny. Dans ces deux textes, il n'y a pas de crypte. L'action dramatique repose sur la révélation simple, dans une dramaturgie d'inspiration classique, bien en deçà des expériences esthétiques qu'avait tentées Loranger dans ses dernières pièces (je pense à Double Jeu et à Medium saignant).

Le premier monologue s'énonce le jour du centième anniversaire de naissance du personnage. 1978 , dit le texte, précisément créé cette annéelà, mais une note prévient que "L'année de naissance de la centenaire change selon l'année de production du texte. Elle a cent ans cette annéelà " (p. 9). La construction, ingénieuse à l'origine, est devenue problématique avec le temps: comment peut-on encore prétendre, si l'on est né en 1901 ou en 1902, que "la plupart de mes oncles avaient participé "à la rébellion de 1837? Reste néanmoins le récit d'une autobiographie fictive, l'histoire d'une jeune bourgeoise née dans une grande famille de Maska, contrainte à des règles d'étiquette venues d'un autre âge, bien que sa famille soit "indigne", rebelle et anticléricale. Le personnage porte en elle-même la mémoire de ces traits de caractères: elle sera rebelle, elle aussi, mais contre l'ordre patriarcal. Mariée contre sa volonté à 17 ans, mère de cinq enfants à 22 , elle se rebelle une première fois contre son "devoir" conjugal et s'enferme loin du monde pendant plusieurs années.
Elle doit néanmoins à son mari, solidaire malgré tout, de n'avoir jamais été internée par sa famille. À cette hiérarchie patriarcale, le personnage oppose la figure du grand-père, le seul être qu'elle aime vraiment et qui lui a légué à la fois la conscience réelle du monde et une faculté de prémonition. Avec cet homme, elle aura une liaison incestueuse qui durera quatre années, jusqu'à ce que la mort les sépare. "Si je la raconte aujourd'hui [mon histoire d'amour] pour la première fois, c'est que votre époque me parait plus capable que la mienne de comprendre certains des phénomènes qui en font partie intégrante, plus capable aussi d'accepter les êtres tels qu'ils sont." (p. 26) Plus qu'une histoire singulière, La dame de cent ans propose une relecture de l'histoire. C'est toute la tradition bourgeoise, que d'aucuns prétendent progressiste, parce que libérale, qui se trouve ici dénoncée.

Quant à Diogène, un ermite, il vient tout juste d'enterrer un homme, décédé sur le pas de sa porte, mais venu dans le nord par crainte d'une guerre atomique. Depuis dix ans, Diogène se terre dans sa cabane, comme un primitif, mais il tient néanmoins son journal, où il consigne sa mémoire et ses souvenirs, en particulier l'histoire de son père alcoolique et de sa femme infidèle. "J'ai dit à tout le monde que je partais comme à l'ordinaire pour un voyage de quelques mois. Reviendrais à l'automne. Jaugeage d'une rivière dans le grand Nord [il est ingénieur]." (p. 44) Peu à peu se dévoile la scène originaire: bien avant les deux autres événements, il avait découvert sa mère dans les bras du père de son ami Francis et l'avait 
entendue dire: "Je voudrais m'en aller [...] M'en aller loin d'ici. [...] Partir avec toi! Ne plus jamais revenir!" (p. 48) L'image de sa mère, vivant une vie de femme et non seulement une vie d'épouse brimée ou de mère, l'a brisé. Ce jour-là il n'a pas pu crier, mais il admet que c'est ce cri "Qui sort enfin aujourd'hui avec un retard de vingt-cinq ans"(p. 48). Lui, qui avait décidé alors de ne jamais retourner parmi les hommes, se met à douter: "Et si c'était vrai? Si j'étais vraiment le dernier être vivant sur la planète?" (p. 53) Une telle question change toute la perspective de son isolement, puisqu'il était en fait arrivé là pour emmerder les autres; si les autres n'y sont plus, son isolement ne sert à rien. La solitude change de valeur. Il doit donc rentrer et trouver une nouvelle vie. "J'irai vers le nord. Je suis sûr d'y rencontrer des Esquimaux. "(p. 56) Au dernier moment, il opte pour le sud.

Alors que Diogène renvoie indéniablement à une vision existentialiste du monde (comme Matbieu, l'unique roman de l'auteure), La dame de cent ans est tout en demi-teintes, et imprégnée, ne serait-ce que dans son pro- pos, d'une vision plus intimiste. La mise en situation rappelle le discours de la mémoire, fût-elle bourgeoise et libérale, discours présenté ici comme tout aussi éteignoir que la chape de religion à laquelle il prétendait échapper. Dernier texte de Françoise Loranger, La dame de cent ans doit donc être lu comme une sorte de testament littéraire, comme une réflexion rétrospective sur l'impossible activité créatrice de son temps et sur le prix qu'il lui a fallu payer pour y parvenir néanmoins.

1. Normand Chaurette, Stabat Mater II, Montréal, Leméac/Actes Sud-Papiers, 1999. 53 p. Sur la structure musicale de la pièce, on lira f'article de Denyse Noreau, . Le Stabat Mater II, un oratorio de la douleur ", Voix et images, $\mathrm{n}^{\circ} 75$. printemps 2000 , p. 471-485.

2. Id., Prouincetou'n Playbouse, juillet 1919, jalais 19 ans, Montréal, Leméac, 1981. p. 79.

3. Idl. Le petit Köchel, Montréal, Leméac/Actes Sud-Papiers, 2000, $51 \mathrm{p}$.

4. Gaétan Soucy, Catoblépas. Théâtre, Montréal. Boréal, 2001, 100 p. Suivi d'un entretien accordé à Stéphanie Jasmin.

5. Stefan P'senak, La fuite comme un voyage, Ottawa, Le Nordir, coll. "Théatre. Rappels", 2001, 97 p.

6. Françoise Loranger, La dame de cent ans, suivi de Diogène 1960. Thêâtre, Montréal, Lanctôt éditeur, 2000, 70 p. 